\title{
Acute Encephalopathy
}

National Cancer Institute

\section{Source}

National Cancer Institute. Acute Encephalopathy. NCI Thesaurus. Code C122815.

A life-threatening disorder characterized by delirium, seizures, and neuromuscular changes. 